\title{
Neooccultibambusa jonesii, a novel taxon within Occultibambusaceae
}

\author{
Jayasiri SC ${ }^{1,2}$, Hyde $\mathrm{KD}^{2,3}$ Jeewon $\mathrm{R}^{4}$, Bhat $\mathrm{JD}^{5}$, Camporesi $\mathrm{E}^{6}$ and Kang $\mathrm{JC}^{1 *}$ \\ ${ }^{1}$ Engineering Research Center of Southwest Bio-Pharmaceutical Resources, Ministry of Education, Guizhou \\ University, Guiyang 550025, Guizhou Province, China \\ ${ }^{2}$ Center of Excellence in Fungal Research, Mae Fah Luang University, Chiang Rai 57100, Thailand \\ ${ }^{3}$ World Agro forestry Centre East and Central Asia Office, 132 Lanhei Road, Kunming 650201, China \\ ${ }^{4}$ Department of Health Sciences, Faculty of Science, University of Mauritius, Reduit, Mauritius \\ ${ }^{5}$ Formerly, Department of Botany, Goa University, Goa, India; No. 128/1-J, Azad Housing Society, Curca, P.O. \\ Goa Velha, 403108, India \\ ${ }^{6}$ A.M.B. GruppoMicologicoForlivese "Antonio Cicognani", Via Roma 18, Forli, Italy; A.M.B. CircoloMicologico \\ "Giovanni Carini”, C.P. 314, Brescia, Italy; Società per gliStudiNaturalisticidella Romagna, C.P. 144, \\ Bagnacavallo (RA), Italy
}

Jayasiri SC, Hyde KD, Jeewon R, Bhat JD, Camporesi E, Kang JC 2016 - Neooccultibambusa jonesii, a novel taxon within Occultibambusaceae. Mycosphere 7 (9) 1458-1472, Doi 10.5943/mycosphere/7/9/17

\begin{abstract}
A new species of Neooccultibambusa, $N$. jonesii, is introduced from Ammophila arenaria collected in Italy. The novel taxon is characterized by immersed to semi-immersed ascomata in fissures of bark, a two-layered peridium, hyaline to brown ascospores and in producing chlamydospores in culture; these characters are typical of Neooccultibambusa. Multigene phylogenies based on analysis of 28S, $18 \mathrm{~S}$ and RPB2 sequence data, indicate that $N$. jonesii belongs in the family Occultibambusaceae and is closely related to $N$. chiangraiensis, but is phylogenetically distinct. Neooccultibambusa jonesii can be differentiated from $N$. chiangraiensis based on the number of septa, the larger and mature dark brown ascospores and in lacking a mucilaginous sheath. We provide a description and photoplates of the new species and compare it with related species, together with phylogenetic inferences based on combined sequence data.
\end{abstract}

Keywords - chlamydospores - Dothideomycetes - monocotyledons - RPB2-rDNA phylogeny - grass

\section{Introduction}

The family Occultibambusaceae was introduced by Dai et al. (2017) with Occultibambusa as the type genus. The family comprises four genera including Occultibambusa, Seriascoma and Versicolorisporium (Dai et al. 2017) and Neooccultibambusa (Doilom et al.

Submitted 18 November 2016, Accepted 23 December 2016, Published 28 December 2016 
2017). These taxa are saprobic on monocotyledons (Dai et al. 2017), such as bamboo, but also have been found on hardwoods, such as teak (Tectona grandis, Doilom et al. 2017).

The grass family (Poaceae, or Gramineae) is one of the most economically important plant families (Soreng et al. 2015). It provides human food in the form of cereals (for example, wheat, rice, barley, oats, millet, maize, sorghum) and sugar (sugar cane) (Raven \& Johnson 1995, Sarwar et al. 2013). In addition, grasses feed cattle, provide the basis for most alcoholic drinks, as well as building materials (bamboo), thatch, and straw (Sarwar et al. 2013). A number of grasses yield essential oils (lemongrass) and raw materials for cosmetics (oats) (Paul \& Julie 2003). The fungi on grasses are also thought be induce respiratory diseases (Purahong \& Hyde 2011). Considering the importance of grasses, it is paramount that we understand the fungi that grow on grasses (Wong \& Hyde 2001). Although there have been many minor studies on graminicolous fungi (Wong \& Hyde 2001), it is only recently that their study has linked morphology and phylogeny (Dai et al. 2012, 2014a, b, c, Liu et al. 2011, 2012, 2014, 2015, Phookamsak et al. 2014, Wijayawardene et al. 2014).

Species of Occultibambusaceae are characterized by immersed, solitary to gregarious ascomata with black ostioles, broadly cylindrical to clavate, bitunicate asci, cellular pseudoparaphyses and broad-fusiform, hyaline to dark brown ascospores, with 1-3 septa. The asexual morphs generally are coelomycetous and morphologically diverse (Hatakeyama et al. 2008, Dai et al. 2017). In the present article, a novel Neooccultibambusa species differing from $N$. chiangraiensis, the generic type, is introduced based on phenotypic characteristics and supported with phylogenies generated from DNA sequence analyses.

\section{Material and methods}

Sample collection and specimen examination

Fresh materials from dead culms of Ammophila arenaria (Poaceae), collected in the Province of Ravenna, Italy in February 2016, were observed using a Motic SMZ 168 Series microscope. Hand sections of fruiting structures were mounted in water for microscopic studies and photomicrography. The fungus was examined with a Nikon ECLIPSE 80i compound microscope and photographed with a Canon 450D digital camera connected to the microscope. Measurements were made with the Tarosoft (R) Image Frame Work program and images used for the Figures were processed with Adobe Photoshop CS6 Extended version 10.0 software (Adobe Systems, USA). Isolations were carried from single ascospores, following a modified method of Chomnunti et al. (2014).

Voucher specimens are deposited in the herbarium of Mae Fah Luang University (Herb. MFLU) and the living cultures are deposited in culture collection of Mae Fah Luang University (MFLUCC), Thailand and Culture Collection of Kunming Institute of Botany (KUMCC) [the latter under Material Transfer Agreement (MTA)]. Faces of fungi and IF numbers are registered as in Jayasiri et al. (2015) and Index Fungorum (2016). New species establishment are based on recommendations as outlined by Jeewon and Hyde (2016).

\section{DNA extraction, PCR amplification and sequencing}

Genomic DNA was extracted from the growing mycelium after 14 days grown on MEA at $18^{\circ} \mathrm{C}$ using the Biospin Fungus Genomic DNA Extraction Kit (BioFlux®) following the manufacturer's protocol (Hangzhou, P.R. China). DNA amplifications were performed by Polymerase Chain Reaction (PCR). The partial large subunit nuclear rDNA (LSU) was amplified with primer pairs LROR and LR5 (Vilgalys and Hester, 1990). The small subunit nuclear rDNA 
(SSU) was amplified with primer pairs NS1 and NS4 (White et al. 1990). The RNA polymerase II second largest subunit (RPB2) gene was amplified with primers fRPB2 and fRPB2-7cR (Carbone and Kohn 1999, Liu et al. 1999, Sung et al. 2007).

The amplification procedure was carried in a $50 \mu 1$ reaction volume containing $2 \mu 1$ DNA, $25 \mu \mathrm{l}$ PCR mix, $19 \mu \mathrm{l}$ distilled water $2 \mu \mathrm{l}$ of each primer. The PCR reactions for amplification of LSU, SSU and RPB2 were performed under standard conditions (White et al. 1990). Purification and sequencing of PCR products were carried at Shanghai Sangon Biological Engineering Technology and Services Co. (China). Newly generated sequences are deposited at NCBI GenBank under the accession numbers provided in Table 1.

Sequence alignment and phylogenetic analysis

LSU, SSU and RPB2 sequence data were verified before further analyses. All introns and exons were aligned individually. Ambiguously aligned regions with many leading or trailing gaps were excluded in alignments prior to tree building. Multiple sequence alignments were produced with MAFFT v. 6.864b (Katoh \& Standley 2013) and further improved manually where necessary and datasets analysed under different optimality criteria as outlined by Jeewon et al $(2002,2003,2013)$. The final phylogenetic tree used to infer the taxonomic placement of our new taxon was generated based on DNA sequence analyses of a concatenated dataset of LSU, SSU and RPB2.

Phylogenetic analyses were performed by using RAxML for maximum likelihood, PAUP v. 4.0 b10 (Swofford 2002) for maximum parsimony and MrBayes v. 3.2 (Ronquist et al. 2011) for Bayesian analyses. Maximum-parsimony analysis was carried in order to obtain the most parsimonious tree. Trees were inferred using the heuristic search option with 1000 random sequence additions. Descriptive tree statistics for parsimony (Tree Length [TL], Consistency Index [CI], Retention Index [RI], Relative Consistency Index [RC] and Homoplasy Index [HI] were calculated for trees generated under different optimality criteria. Kishino-Hasegawa tests (KHT) (Kishino and Hasegawa 1989) were performed in order to determine whether trees were significantly different. Maximum parsimony bootstrap values equal or greater than $60 \%$ are given near to each node (Fig. 1). A maximum likelihood analysis was performed at the CIPRES webportal (Miller et al. 2010) using RAxML-HPC2 on XSEDE (v 8.2.8) with default parameters and bootstrapping with 1000 replicates (Stamatakis 2006, Stamatakis et al. 2008). Maximum likelihood bootstrap values (MLBS) equal or greater than $60 \%$ are given at each node (Fig. 1).

An appropriate model of evolution was selected by using MrModeltest 2.2 (Nylander 2004) for each gene partition. Posterior probabilities (Rannala \&Yang 1996, Zhaxybayeva \& Gogarten 2002) were determined by Markov Chain Monte Carlo sampling (MCMC) in MrBayes v. 3.2 (Ronquist et al. 2011). The Bayesian inference was conducted under different models for each partition of the matrix as evaluated by MrModeltest 2.2 (Nylander 2004). Six simultaneous Markov chains were run for 100000000 generations and every 1000th generation a tree was sampled. MCMC heated chain was set with a "temperature" value of 0.15 . The distribution of log-likelihood scores was examined to determine stationary phase for each search and to decide if extra runs were required to attain convergence, using the program Tracer 1.5 (Rambaut \& Drummond 2007). All sampled topologies beneath the asymptote (25\%) were discarded as part of a burn-in procedure and the remaining trees were used for calculating posterior probabilities in the majority rule consensus tree. Bayesian Posterior Probabilities (BPP) equal or greater than 0.90 is given near to each node (Fig. 1). Phylogenetic trees and data files were viewed FigTree v. 1.4 (Rambaut \& Drummond 2008). Novel sequences generated in this study are deposited in 
Table 1 Taxa used in the phylogenetic analysis and GenBank accession numbers (LSU, SSU and RPB2 sequence data) and species. The newly generated sequences are indicated in bold.

\begin{tabular}{|c|c|c|c|c|c|}
\hline \multirow[t]{2}{*}{ Species name } & \multirow[t]{2}{*}{ Family } & \multirow[t]{2}{*}{ Culture no. } & \multicolumn{3}{|c|}{ Gene Bank accession no. } \\
\hline & & & LSU & SSU & RPB2 \\
\hline Aigialus grandis & Aigialaceae & BCC 18419 & GU479774 & GU479738 & GU479813 \\
\hline Aigialus mangrovis & Aigialaceae & BCC 33564 & GU479777 & GU479742 & GU479816 \\
\hline $\begin{array}{l}\text { Anteaglonium } \\
\text { globosum }\end{array}$ & Anteagloniaceae & SMH 5283 & GQ221911 & NA & NA \\
\hline $\begin{array}{l}\text { Anteaglonium } \\
\text { latirostrum }\end{array}$ & Anteagloniaceae & GKM 1119 & GQ221874 & NA & NA \\
\hline $\begin{array}{l}\text { Anteaglonium } \\
\text { parvulum }\end{array}$ & Anteagloniaceae & SMH 5223 & GQ221909 & NA & NA \\
\hline $\begin{array}{l}\text { Anteaglonium } \\
\text { abbreviatum }\end{array}$ & Anteagloniaceae & ANM 925a & GQ221877 & NA & NA \\
\hline $\begin{array}{l}\text { Arthopyrenia } \\
\text { salicis }\end{array}$ & Roussoellaceae & $\begin{array}{l}\text { CBMAI } \\
1330\end{array}$ & JN903536 & NA & NA \\
\hline $\begin{array}{l}\text { Arthopyrenia } \\
\text { salicis }\end{array}$ & Roussoellaceae & CBS 368.94 & AY538339 & AY538333 & GU371814 \\
\hline $\begin{array}{l}\text { Bambusicola } \\
\text { massarinia }\end{array}$ & Bambusicolaceae & $\begin{array}{l}\text { MFLUCC } \\
11-0389^{\mathrm{T}}\end{array}$ & JX442037 & JX442041 & KP761716 \\
\hline $\begin{array}{l}\text { Bambusicola } \\
\text { splendida }\end{array}$ & Bambusicolaceae & $\begin{array}{l}\text { MFLUCC } \\
11-0439^{\mathrm{T}}\end{array}$ & JX442038 & JX442042 & NA \\
\hline $\begin{array}{l}\text { Biatriospora } \\
\text { marina }\end{array}$ & Biatriosporaceae & CY 1228 & GQ925848 & GQ925835 & GU479823 \\
\hline Biatriospora sp. & Biatriosporaceae & E 11301D & LN626685 & LN626675 & LN626663 \\
\hline $\begin{array}{l}\text { Biatriospora } \\
\text { mackinnonii }\end{array}$ & Biatriosporaceae & CBS 110022 & KF015609 & GQ387553 & KF015704 \\
\hline $\begin{array}{l}\text { Biatriospora } \\
\text { mackinnonii }\end{array}$ & Biatriosporaceae & CBS 674.75 & KF015612 & GQ387552 & KF015703 \\
\hline $\begin{array}{l}\text { Byssosphaeria } \\
\text { salebrosa }\end{array}$ & Melanommataceae & SMH 2387 & NA & NA & GU385162 \\
\hline $\begin{array}{l}\text { Corynespora } \\
\text { cassiicola }\end{array}$ & Corynesporaceae & CBS 100822 & GU301808 & GU296144 & GU357772 \\
\hline $\begin{array}{l}\text { Corynespora } \\
\text { smithii }\end{array}$ & Corynesporaceae & CABI 5649b & GU323201 & NA & GU371804 \\
\hline $\begin{array}{l}\text { Decaisnella } \\
\text { formosa }\end{array}$ & Lophiostomataceae & BCC 25617 & GQ925847 & GQ925834 & GU479824 \\
\hline $\begin{array}{l}\text { Delitschia } \\
\text { chaetomioides }\end{array}$ & Delitschiaceae & SMH 3253.2 & GU390656 & NA & NA \\
\hline Delitschia winteri & Delitschiaceae & $\begin{array}{l}\text { AFTOL-ID } \\
1599\end{array}$ & DQ678077 & DQ678026 & DQ677975 \\
\hline $\begin{array}{l}\text { Dendryphion } \\
\text { europaeum }\end{array}$ & Torulaceae & CРC 22943 & KJ869203 & NA & NA \\
\hline $\begin{array}{l}\text { Dendryphion } \\
\text { europaeum }\end{array}$ & Torulaceae & СРC 23231 & KJ869202 & NA & NA \\
\hline
\end{tabular}


Table 1 continued

\begin{tabular}{|c|c|c|c|c|c|}
\hline \multirow[t]{2}{*}{ Species name } & \multirow[t]{2}{*}{ Family } & \multirow[t]{2}{*}{ Culture no. } & \multicolumn{3}{|c|}{ Gene Bank accession no. } \\
\hline & & & LSU & SSU & RPB2 \\
\hline $\begin{array}{l}\text { Elongatopedicellata } \\
\text { lignicola }\end{array}$ & Roussoellaceae & $\begin{array}{l}\text { MFLUCC } \\
15-0642^{\mathrm{T}}\end{array}$ & NA & NA & NA \\
\hline $\begin{array}{l}\text { Fissuroma } \\
\text { maculans }\end{array}$ & Aigialaceae & $\begin{array}{l}\text { MFLUCC10- } \\
0886\end{array}$ & NG042598 & JN846734 & NA \\
\hline $\begin{array}{l}\text { Halomassarina } \\
\text { thalassiae }\end{array}$ & Trematosphaeriaceae & BCC 17054 & GQ925849 & GQ925842 & NA \\
\hline $\begin{array}{l}\text { Halomassarina } \\
\text { thalassiae }\end{array}$ & Trematosphaeriaceae & BCC 17055 & GQ925850 & GQ925843 & NA \\
\hline $\begin{array}{l}\text { Halotthia } \\
\text { posidoniae }\end{array}$ & Halotthiaceae & BBH 22481 & GU479786 & GU479752 & NA \\
\hline Helicascus nypae & Morosphaeriaceae & ВСС 36752 & GU479789 & GU479755 & GU479827 \\
\hline $\begin{array}{l}\text { Herpotrichia } \\
\text { macrotricha }\end{array}$ & Melanommataceae & GKM 196N & GU385176 & NA & NA \\
\hline $\begin{array}{l}\text { Hysterium } \\
\text { angustatum }\end{array}$ & Hysteriaceae & CBS 236.34 & NA & GU397359 & FJ161117 \\
\hline $\begin{array}{l}\text { Keissleriella } \\
\text { cladophila }\end{array}$ & Lentitheciaceae & CBS 104.55 & JX681090 & GU296155 & GU371735 \\
\hline $\begin{array}{l}\text { Lentithecium } \\
\text { fluviatile }\end{array}$ & Lentitheciaceae & CBS 122367 & GU301825 & GU296158 & NA \\
\hline $\begin{array}{l}\text { Leptosphaeria } \\
\text { biglobosa }\end{array}$ & Leptosphaeriaceae & CBS 303.51 & GU301826 & NA & NA \\
\hline $\begin{array}{l}\text { Leptosphaeria } \\
\text { doliolum }\end{array}$ & Leptosphaeriaceae & CBS 505.75 & GQ387576 & GQ387515 & KT389640 \\
\hline $\begin{array}{l}\text { Longiostiolum } \\
\text { tectonae }\end{array}$ & & $\begin{array}{l}\text { MFLUCC } \\
12-0562\end{array}$ & KU764700 & KU712459 & NA \\
\hline $\begin{array}{l}\text { Lophiostoma } \\
\text { macrostomoides }\end{array}$ & Lophiostomataceae & GKM 1033 & GU385190 & NA & NA \\
\hline $\begin{array}{l}\text { Lophiotrema } \\
\text { lignicola }\end{array}$ & Lophiotremataceae & CBS122364 ${ }^{\mathrm{T}}$ & GU301836 & GU296166 & FJ795462 \\
\hline $\begin{array}{l}\text { Lophiotrema } \\
\text { nucula }\end{array}$ & Lophiotremataceae & CBS 627.86 & GU301837 & GU296167 & FJ795463 \\
\hline Massaria inquinans & Massariaceae & CBS122369 & GU456322 & GU456300 & NA \\
\hline $\begin{array}{l}\text { Massarina } \\
\text { velataspora }\end{array}$ & Morosphaeriaceae & BCC 17059 & GQ925852 & GQ925841 & $\mathrm{NA}$ \\
\hline $\begin{array}{l}\text { Mauritiana } \\
\text { rhizophorae }\end{array}$ & Halotthiaceae & BCC 28866 & GU371824 & GU371832 & $\mathrm{NA}$ \\
\hline $\begin{array}{l}\text { Melanommapulvis } \\
\text { pyrius }\end{array}$ & Melanommataceae & CBS 124080 & GU456323 & GU456302 & GU456350 \\
\hline $\begin{array}{l}\text { Monotosporella } \\
\text { tuberculata }\end{array}$ & Melanommataceae & CBS 256.84 & GU301851 & NA & NA \\
\hline $\begin{array}{l}\text { Montagnula } \\
\text { opulenta }\end{array}$ & Didymosphaeriaceae & CBS 168.34 & NG027581 & NA & NA \\
\hline $\begin{array}{l}\text { Morosphaeria } \\
\text { ramunculicola }\end{array}$ & Morosphaeriaceae & JK 5304B & GU479794 & GU479760 & GU479831 \\
\hline
\end{tabular}


Table 1 continued

\begin{tabular}{|c|c|c|c|c|c|}
\hline \multirow[t]{2}{*}{ Species name } & \multirow[t]{2}{*}{ Family } & \multirow[t]{2}{*}{ Culture no. } & \multicolumn{3}{|c|}{ Gene Bank accession no. } \\
\hline & & & LSU & SSU & RPB2 \\
\hline $\begin{array}{l}\text { Neooccultibambusa } \\
\text { chiangraiensis }\end{array}$ & Occultibambusaceae & $\begin{array}{l}\text { MFLUCC } \\
12-0559^{\mathrm{T}} \\
\end{array}$ & KU764699 & KU712458 & NA \\
\hline $\begin{array}{l}\text { Neooccultibambusa } \\
\text { jonesii }\end{array}$ & Occultibambusaceae & $\begin{array}{l}\text { MFLUCC } \\
160643 \\
\end{array}$ & KY111437 & KY111438 & NA \\
\hline Neooccultibambusa sp. & Occultibambusaceae & $\begin{array}{l}\text { MFLUCC } \\
12-0564\end{array}$ & NA & NA & NA \\
\hline $\begin{array}{l}\text { Neoroussoella } \\
\text { bambusea }\end{array}$ & Roussoellaceae & $\begin{array}{l}\text { MFLUCC } \\
11-0124^{\mathrm{T}} \\
\end{array}$ & KJ474839 & NA & KJ474856 \\
\hline $\begin{array}{l}\text { Occultibambusa } \\
\text { bambusae }\end{array}$ & Occultibambusaceae & $\begin{array}{l}\text { MFLUCC } \\
11-0394\end{array}$ & KU863113 & NA & KU940171 \\
\hline $\begin{array}{l}\text { Occultibambusa } \\
\text { bambusae }\end{array}$ & Occultibambusaceae & $\begin{array}{l}\text { MFLUCC } \\
13-0855\end{array}$ & KU863112 & NA & KU940170 \\
\hline $\begin{array}{l}\text { Occultibambusa } \\
\text { fusispora }\end{array}$ & Occultibambusaceae & $\begin{array}{l}\text { MFLUCC } \\
11-0127 \\
\end{array}$ & KU863114 & NA & KU940172 \\
\hline Occultibambusa pustula & Occultibambusaceae & $\begin{array}{l}\text { MFLUCC } \\
11-0502 \\
\end{array}$ & KU863115 & NA & NA \\
\hline $\begin{array}{l}\text { Ophiosphaerella } \\
\text { sasicola }\end{array}$ & Lentitheciaceae & KT 1706 & AB524599 & AB524458 & AB539098 \\
\hline $\begin{array}{l}\text { Paraconiothyrium } \\
\text { minitans }\end{array}$ & Didymosphaeriaceae & CBS 122788 & EU754173 & EU754074 & GU357807 \\
\hline $\begin{array}{l}\text { Paradictyoarthrinium } \\
\text { diffractum }\end{array}$ & Paradictyoarthriniaceae & $\begin{array}{l}\text { MFLUCC } \\
12-0557\end{array}$ & KP744497 & NA & NA \\
\hline $\begin{array}{l}\text { Paradictyoarthrinium } \\
\text { diffractum }\end{array}$ & Paradictyoarthriniaceae & $\begin{array}{l}\text { MFLUCC } \\
13-0466 \\
\end{array}$ & KP744498 & KP753960 & NA \\
\hline $\begin{array}{l}\text { Paradictyoarthrinium } \\
\text { tectonicola }\end{array}$ & Paradictyoarthriniaceae & $\begin{array}{l}\text { MFLUCC } \\
12-0556 \\
\end{array}$ & KP744499 & NA & NA \\
\hline $\begin{array}{l}\text { Paradictyoarthrinium } \\
\text { tectonicola }\end{array}$ & Paradictyoarthriniaceae & $\begin{array}{l}\text { MFLUCC } \\
13-0465^{\mathrm{T}} \\
\end{array}$ & KP744500 & KP753961 & NA \\
\hline Pleomassaria siparia & Pleomassariaceae & $\begin{array}{l}\text { AFTOL-ID } \\
1600\end{array}$ & DQ678078 & DQ678027 & DQ677976 \\
\hline Preussia funiculata & Sporormiaceae & CBS 659.74 & GU301864 & GU296187 & GU371799 \\
\hline Preussia minima & Sporormiaceae & $\begin{array}{l}\text { AFTOL-ID } \\
1256 \\
\end{array}$ & DQ678056 & DQ678003 & DQ677950 \\
\hline Prosthemium stellare & Pleomassariaceae & $\begin{array}{l}\text { VM } \\
20050611 \\
\end{array}$ & AB553781 & AB553650 & NA \\
\hline $\begin{array}{l}\text { Pseudocoleodictyospora } \\
\text { sukhothaiensis }\end{array}$ & Pseudocoleodictyosporaceae & $\begin{array}{l}\text { MFLUCC } \\
12-0554^{\mathrm{T}} \\
\end{array}$ & KU764710 & KU712471 & KU712493 \\
\hline $\begin{array}{l}\text { Pseudocoleodictyospora } \\
\text { tectonae }\end{array}$ & Pseudocoleodictyosporaceae & $\begin{array}{l}\text { MFLUCC } \\
12-0385^{\mathrm{T}} \\
\end{array}$ & KU764704 & KU712461 & KU712491 \\
\hline $\begin{array}{l}\text { Pseudocoleodictyospora } \\
\text { tectonae }\end{array}$ & Pseudocoleodictyosporaceae & $\begin{array}{l}\text { MFLUCC } \\
12-0387 \\
\end{array}$ & NA & KU712462 & KU712492 \\
\hline $\begin{array}{l}\text { Pseudocoleodictyospora } \\
\text { thailandica }\end{array}$ & Pseudocoleodictyosporaceae & $\begin{array}{l}\text { MFLUCC } \\
12-0565^{\mathrm{T}}\end{array}$ & KU764701 & KU712472 & KU712494 \\
\hline
\end{tabular}


Table 1 continued

\begin{tabular}{|c|c|c|c|c|c|}
\hline \multirow{2}{*}{ Species name } & \multirow{2}{*}{ Family } & \multirow{2}{*}{ Culture no. } & \multicolumn{3}{|c|}{ Gene Bank accession no. } \\
\hline & & & LSU & SSU & RPB2 \\
\hline $\begin{array}{l}\text { Quadricrura } \\
\text { septentrionalis }\end{array}$ & Tetralosphaeriaceae & HHUF 28782 & NG042327 & AB524475 & NA \\
\hline $\begin{array}{l}\text { Roussoella } \\
\text { hysterioides }\end{array}$ & Roussoellaceae & HH 26988 & AB524622 & AB524481 & AB539102 \\
\hline Roussoella nitidula & Roussoellaceae & $\begin{array}{l}\text { MFLUCC 11- } \\
0182^{\mathrm{T}}\end{array}$ & KJ474843 & NA & KJ474859 \\
\hline Roussoella nitidula & Roussoellaceae & $\begin{array}{l}\text { MFLUCC 11- } \\
0634\end{array}$ & KJ474842 & NA & KJ474858 \\
\hline $\begin{array}{l}\text { Roussoella } \\
\text { pustulans }\end{array}$ & Roussoellaceae & KT 1709 & AB524623 & AB524482 & AB539103 \\
\hline $\begin{array}{l}\text { Seriascoma } \\
\text { didymosporum }\end{array}$ & Occultibambusaceae & $\begin{array}{l}\text { MFLUCC 11- } \\
0179\end{array}$ & KU863116 & NA & KU940173 \\
\hline $\begin{array}{l}\text { Seriascoma } \\
\text { didymosporum }\end{array}$ & Occultibambusaceae & $\begin{array}{l}\text { MFLUCC 11- } \\
0194\end{array}$ & KU863117 & NA & KU940174 \\
\hline $\begin{array}{l}\text { Sporidesmium } \\
\text { australiense }\end{array}$ & Roussoellaceae & $\begin{array}{l}\text { HKUCC } \\
10833\end{array}$ & DQ408554 & NA & DQ435080 \\
\hline $\begin{array}{l}\text { Subglobosporium } \\
\text { tectonae }\end{array}$ & Pseudocoleodictyosporaceae & $\begin{array}{l}\text { MFLUCC 12- } \\
0390\end{array}$ & NA & KU712463 & KU712495 \\
\hline $\begin{array}{l}\text { Subglobosporium } \\
\text { tectonae }\end{array}$ & Pseudocoleodictyosporaceae & $\begin{array}{l}\text { MFLUCC 12- } \\
0393^{\mathrm{T}}\end{array}$ & KU764703 & KU712464 & KU712485 \\
\hline $\begin{array}{l}\text { Tetraplosphaeria } \\
\text { sasicola }\end{array}$ & Tetralosphaeriaceae & KT 563 & AB524631 & AB524490 & NA \\
\hline $\begin{array}{l}\text { Thyridaria } \\
\text { rubronotata }\end{array}$ & Thyridariaceae & CBS 419.85 & GU301875 & NA & GU371728 \\
\hline Torula ficus & Torulaceae & CBS 595.96 & KF443385 & KF443387 & KF443395 \\
\hline Torula herbarum & Torulaceae & CBS 111855 & KF443386 & KF443391 & KF443396 \\
\hline Torula herbarum & Torulaceae & CBS 379.58 & CBS 379.58 & KF443388 & NA \\
\hline Torula herbarum & Torulaceae & CPC $24114^{\mathrm{T}}$ & KR873288 & NA & NA \\
\hline Torula hollandica & Torulaceae & CBS 220.69 & KF443384 & KF443389 & KF443393 \\
\hline Torula masonii & Torulaceae & CBS 246.57 & KR873290 & NA & NA \\
\hline Torula masonii & Torulaceae & CBS 245.57 & KR873289 & NA & NA \\
\hline $\begin{array}{l}\text { Trematosphaeria } \\
\text { pertusa }\end{array}$ & Trematosphaeriaceae & CBS 122368 & FJ201990 & FJ201991 & FJ795476 \\
\hline $\begin{array}{l}\text { Versicolorisporium } \\
\text { triseptatum }\end{array}$ & Occultibambusaceae & HHUF:28815 & AB330081 & AB524501 & NA \\
\hline $\begin{array}{l}\text { Westerdykella } \\
\text { ornata }\end{array}$ & Sporormiaceae & CBS 379.55 & GU301880 & GU296208 & GU371803 \\
\hline
\end{tabular}

$\mathrm{T}$ = Type strain.

$\mathrm{NA}=$ not available.

GenBank (Table 1) and the final matrices used for phylogenetic analyses were saved in TreeBASE (www.treebase.org; TB2:S20198).

\section{Results}




\section{Phylogeny}

Irrespective of the criteria used in the analyses of single or combined genes datasets, phylogenies generated were topologically congruent (results not shown). Fig. 1 represents the relationships of our new taxon. This dataset consisted of 2589 characters (LSU-863, SSU-951, RPB2-783) of which 1563 were constant, 179 variable parsimony uninformative characters and 847 parsimony informative characters (32\%) while Hysterium angustatum CBS 236.34 was the outgroup taxon. The parsimony analysis of the data matrix resulted in three equally parsimonious trees and the best tree support values belong to the first tree $(\mathrm{TL}=6000$ steps, $\mathrm{CI}=0.287, \mathrm{RI}=$ $0.598, \mathrm{RC}=0.172$ and $\mathrm{HI}=0.713$ ). The Bayesian analysis resulted in 2438 trees after 2438000 generations. The first 610 trees, representing the burn-in phase of the analyses were discarded, while the remaining trees were used for calculating posterior probabilities in the majority rule consensus tree (split frequency was lower than 0.01). A best scoring RAxML tree resulted with the value of likelihood: -29664.999375. Phylogenetic trees obtained from maximum parsimony, maximum likelihood and Bayesian analysis yielded trees with similar overall topology at the species level and are in agreement with previous studies (Hyde et al. 2013, Dai et al. 2017, Doilom et al. 2017). The strain of Neooccultibambusa jonesii formed a sister clade to Neooccultibambusa sp. with high bootstrap value in Bayesian analysis (0.93BPP) and maximum parsimony (61\%), but lower value in maximum likelihood analysis. Therefore, a new species is introduced to accommodate this taxon within the genus Neooccultibambusa.

\section{Taxonomy}

\section{Neooccultibambusa jonesii Jayasiri, Camporesi \& K.D. Hyde, sp. nov}

Index Fungorum number: IF552518; Facesoffungi number: FoF 02642,

Fig. 2

Etymology: Named in honour of Professor E.B. Gareth Jones for his contributions to mycology.

Holotype: MFLU 16-0871

Saprobic on dead and aerial stems of Ammophila arenaria L. Sexual morph: Ascomata 104-155 $\mu \mathrm{m}$ high $\times 130-160 \mu \mathrm{m}$ diameter $(\bar{x}=128 \times 151 \mu \mathrm{m}, \mathrm{n}=10)$, scattered to gregarious, semi-immersed to densely erumpent, carbonaceous, dark brown, globose to subglobose. Ostiole with a small to large, flat, crest-like apex, with apex composed of pseudoparenchymatous cells. Peridium 16-22 $\mu \mathrm{m}$ thick near the ostiole, with inner layer composed of hyaline cells of textura angularis, outer layer dark brown, 5-6 layers. Hamathecium comprising of 1.3-2 $\mu \mathrm{m}$ diam., septate, hyaline, anastomosing and branched, cellular pseudoparaphyses, embedded in gelatinous matrix between and above the asci. Asci $47-76 \times 8-10 \mu \mathrm{m}$ diameter $(\bar{x}=60 \times 9 \mu \mathrm{m}, \mathrm{n}=20), 8$ spored, bitunicate, fissitunicate, cylindrical to clavate, with furcate pedicel, rounded at apex with a minute ocular chamber. Ascospores 15-20 $\times 2.5-4.5 \mu \mathrm{m}(\bar{x}=17 \times 4 \mu \mathrm{m}, \mathrm{n}=30)$, uniseriate to biseriate, narrowly fusiform with acute ends, 1-septate, 2 septa in germinated spores (Fig 3a), constricted at the septa, with distinct oil drops in each cell, smooth-walled, hyaline, pale brown to dark brown. Asexual morph: Colonies circular, floccose, dark brown to black, with smooth, circular margin, $20 \mathrm{~mm}$ diameter in 3 weeks, Mycelium 2-3.5 $\mu \mathrm{m}$ wide, aerial to immersed, composed of pale brown to dark brown, septate, branched hyphae, slightly constricted at septa, walls with melanin deposits. Conidiophores macronematous, prostrate, flexuous, septate, branched, dark brown, semi- to immersed. Conidiogenous cells gangliar-type, terminal or intercalary. Conidia 10-14 $(-16) \times 8-14(-17)(\bar{x}=13 \times 13 \mu \mathrm{m}, \mathrm{n}=20)$, subglobose to globose, unicellular, initially subhyaline, becoming pale to dark brown at maturity, thick-walled, smooth, sometimes wall collapsing. 


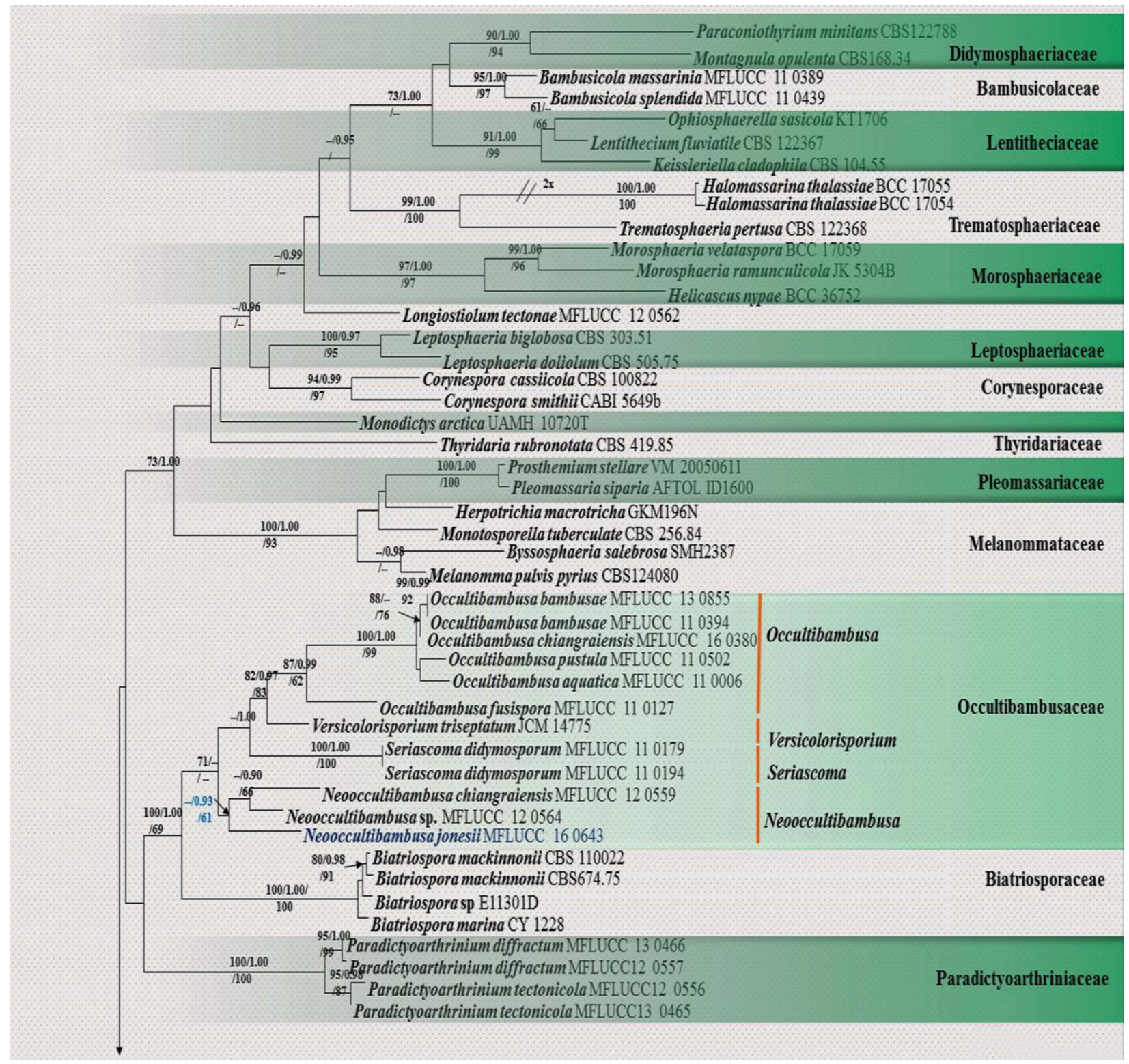

Fig. 1 Phylogram generated from maximum likelihood analysis (RAxML) based on combined LSU, SSU and RPB2 sequence data from species of Pleosporales. The tree is rooted to Hysterium angustatum CBS 236.34. Maximum Likelihood bootstrap values (MLBS) $\geq 60 \%$, Bayesian posterior probabilities (BPP) $\geq 0.90$, and Maximum Parsimony (MP) $\geq 60 \%$ are given at the nodes respectively. Some branches were shortened to fit the page - these are indicated by two diagonal lines with the number of times a branch was shortened indicated next to the lines. The new isolate is in blue.

Culture characteristics - Ascospores germinating on MEA within 24 hours. Germ tubes produced at both ends of ascospores and can see two septate stage of ascospore. Colonies on MEA reaching $20 \mathrm{~mm}$ diameter after 3 weeks, circular, convex or dome-shaped, cottony, aerial 
in the center, immersed at the edge, edge entire, dense, with colonies becoming brownish grey after 3 weeks.

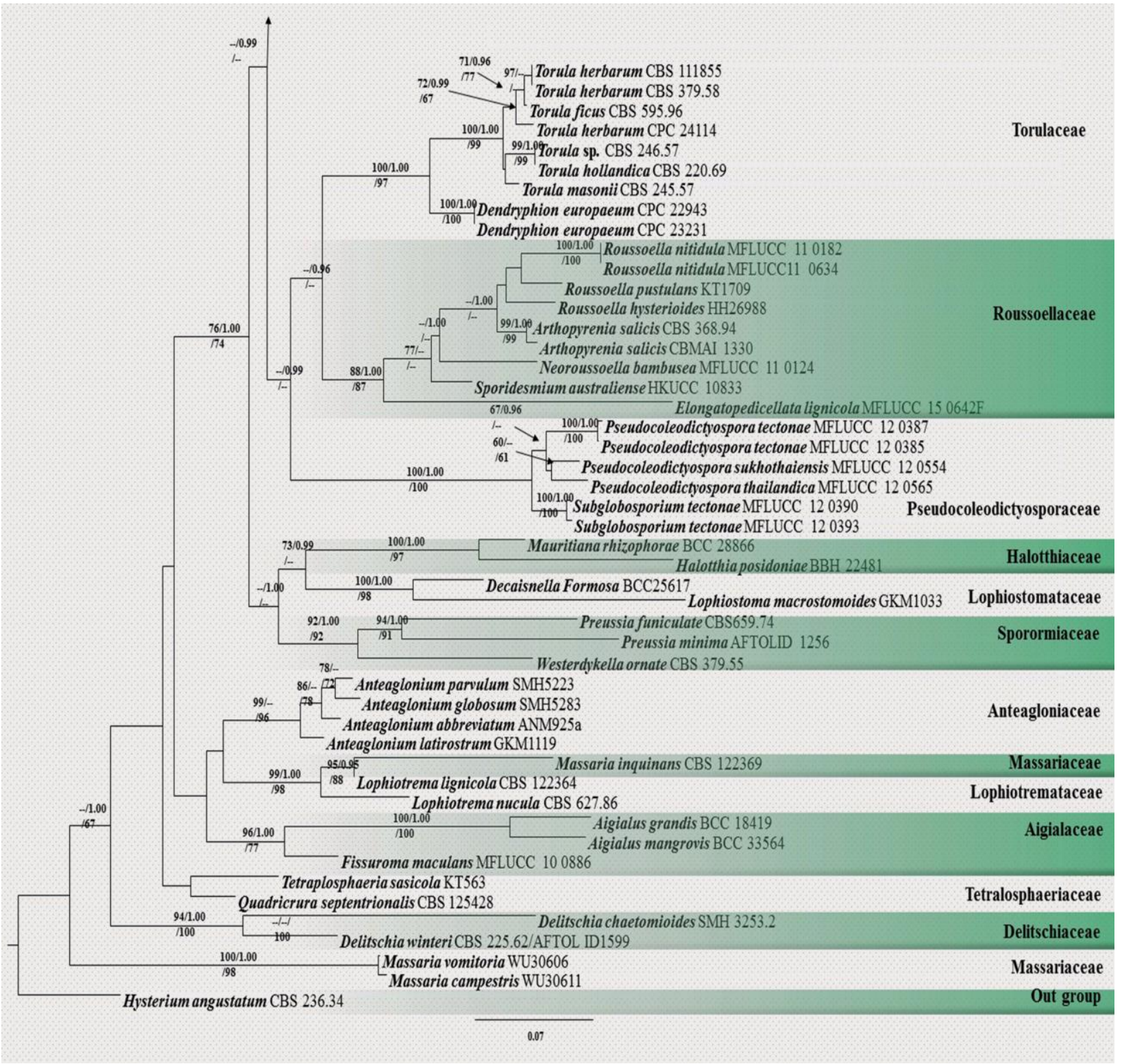

Fig 1 continued.

Material examined - ITALY, Province of Ravenna, Lido di Dante, dead and aerial stem of Ammophila arenaria (Poaceae), 9 February 2016, Erio Camporesi, IT 2813 (MFLU 16-0871, holotype); (isotype in KUN), ex-type living culture, MFLUCC 16 0643; KUMCC 16-0110.

\section{Discussion}

Neooccultibambusa, is represented by the generic type, $N$. chiangraiensis, and is similar to Occultibambusa in having dark brown, fusiform ascospores, surrounded by a gelatinous sheath. However, Neooccultibambusa produces cylindrical to subcylindrical asci and ascospores with 1-3 transverse septa and Occultibambusa produces broadly cylindrical to clavate asci and 

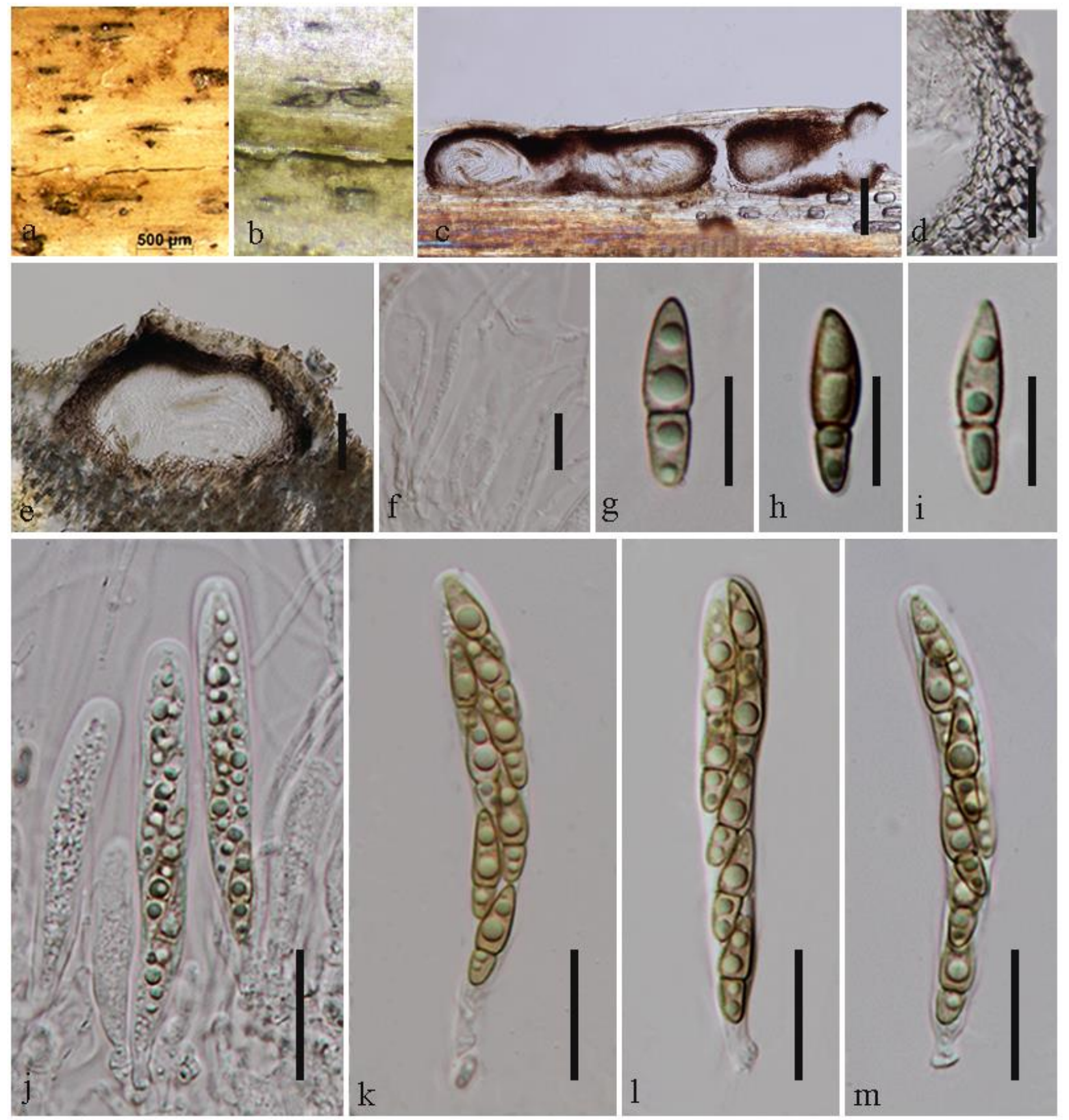

Fig. 2 - Neooccultibambusa jonesii (holotype). a, b Appearance of substrate with ascomata. c, e Sections of ascomata. d Peridium. f Pseudoparaphyses. g-i Ascospores. j-m Asci. Scale bars: c, e $=50 \mu \mathrm{m}, \mathrm{d}=20 \mu \mathrm{m}, \mathrm{f}=50 \mu \mathrm{m}, \mathrm{g}-\mathrm{i}=10 \mu \mathrm{m}, \mathrm{j}-\mathrm{m}=30 \mu \mathrm{m}$.

ascospores with 1 transverse septum. Phylogenetic analyses also separate Neooccultibambusa from Occultibambusa (Dai et al. 2017, Doilom et al. 2017). Currently Neooccultibambusa comprises two taxa, N. chiangraiensis (MFLUCC 12 0559) and an undescribed species, Neooccultibambusa sp. (MFLUCC 12 0564, Dai et al. 2017, Doilom et al. 2017). However, descriptions and illustrations of MFLUCC 12-0564 are not available and therefore $N$. jonesii is 


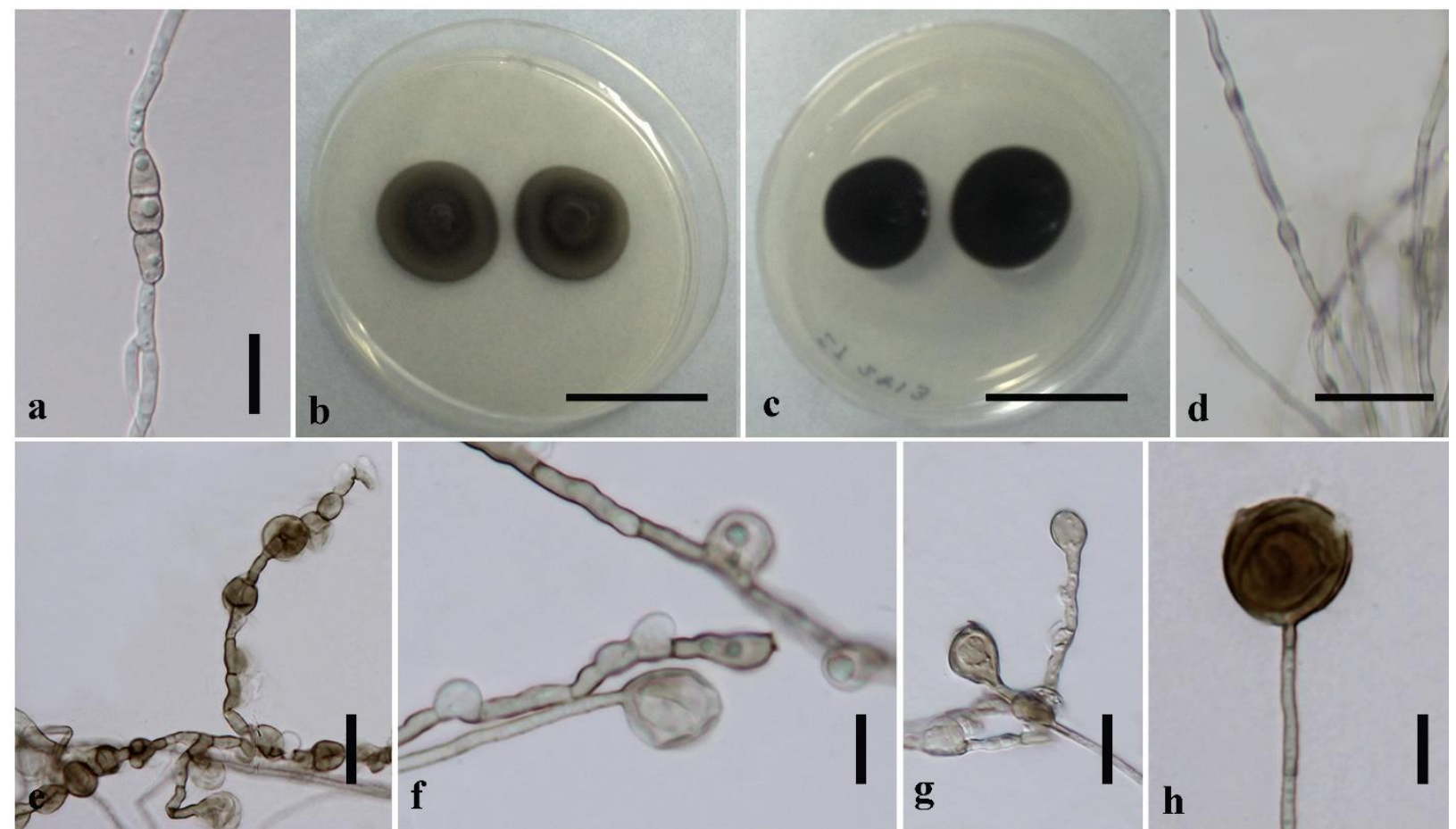

Fig. 3 - Morphology of Neooccultibambusa jonesii on MEA (from MFLUCC 16 0643). a Germinated ascospore in culture. b, c Colonies after 7 days from single spore isolation. d Mycelia with melanin deposits. e-g Conidia produced on hyphae. h Conidium. Scale bars: a, d-h $=10 \mu \mathrm{m}, \mathrm{b}, \mathrm{c}=4 \mathrm{~cm}$.

compared with generic type, $N$. chiangraiensis (Doilom et al. 2017). Neooccultibambusa jonesii differs from $N$. chiangraiensis in having dark brown ascospores with prominent guttules, and smaller ascospores $(15-20 \times 2.5-4.5 \mu \mathrm{m})$. In addition, Neooccultibambusa jonesii can be segregated from $N$. chiangraiensis based on the number of septa (2, versus 1). The morphological differences are also supported by analysis of combined LSU, SSU and RPB2 sequence data. DNA sequence comparison between $N$. chiangraiensis and $N$. jonesii reveals some striking differences among nucleotides that further supports the establishment of the new taxon. In particular, there are 24 and 14 base pair differences in the LSU and SSU regions respectively between these two species.

Ammophila arenaria is a common grass species found in coastal sound dunes in many countries, with 241 associated fungal taxa (Treigienè 2011, Farr \& Rossman 2016). In addition, a number of new species have been described from this host. For example, Peziza fruticosa and Anthostomella ravennica have recently been collected and described from Italy (Hyde et al. 2016). Many Dothideomycetes species in the genera Didymosphaeria, Leptosphaeria, Mycosphaerella, Phaeosphaeria and Pleospora, have been recorded from this host (Farr \& Rossman 2016). Most of these records however, need confirming with molecular data.

\section{Acknowledgements}

Subashini C. Jayasiri is grateful to EB Gareth Jones for his generosity in supporting her $\mathrm{PhD}$ study. All authors would like to acknowledge his significant contributions to mycology, especially in the fields of aquatic and Asian fungi. K.D. Hyde thanks the Chinese Academy of Sciences, [project number 2013T2S003] for the award of Visiting Professorship of Senior 
International Scientists at Kunming Institute of Botany. The authors thank the National Natural Science Foundation of China (Grant No. 31460011) for supporting and funding this research. We thank Guizhou University, China for assistance with the molecular work. We are also grateful to the Mushroom Research Foundation, Chiang Rai, Thailand for support. The authors thank TingChi Wen, Xiaoya Ma and Yuan-Pin Xiao from Guizhou University, China, for help with sequence data analyses and also Mingkwan Doilom from Mae Fah Luang University, Thailand for help with giving sequence data. Rajesh Jeewon thanks Mae Fah Lunag University for the award of a short term Visiting Professor. Subashini C. Jayasiri is grateful to Mr. and (Late) Mrs. Jayasiri, S.P.R.D. Lasantha for their valuable support.

\section{References}

Carbone I, Kohn L. 1999 - A method for designing primer sets for speciation studies in filamentous ascomycetes. Mycologia 91, 553-556.

Chomnunti P, Hongsanan S, Hudson BA, Tian Q et al. 2014 - The sooty moulds. Fungal Diversity 66, 1-36.

Dai DQ, Bahkali AH, Li QR, Bhat DJ et al. 2014c - Vamsapriya (Xylariaceae) re-described, with two new species and molecular sequence data. Cryptogamie Mycologie 35, 339-357.

Dai DQ, Bhat DJ, Liu JK, Chukeatirote E et al. 2012 - Bambusicola, a new genus from bamboo with asexual and sexual morphs. Cryptogamie Mycologie 33, 363-379.

Dai DQ, Phookamsak R, Wijayawardene NN, Li WJ et al. 2017 - Bambusicolous fungi. Fungal Diversity (published online) DOI 10.1007/s13225-016-0367-8

Dai DQ, Wijayawardene NN, Bhat DJ, Chukeatirote E et al. 2014a - Pustulomyces gen. nov. accommodated in Diaporthaceae, Diaporthales, as revealed by morphology and Molecular analyses. Cryptogamie Mycologie 35, 63-72.

Dai DQ, Wijayawardene NN, Bhat DJ, Chukeatirote E et al. 2014b - The Phylogenetic Placement of Eriosporella bambusicola sp. nov. in Capnodiales. Cryptogamie Mycologie 35, 41-49.

Doilom M, Dissanayake AJ, Wanasinghe DN, Boonmee S et al. 2017 - Microfungi on Tectona grandis (teak) in northern Thailand. Fungal Diversity (published online) DOI 10.1007/s13225-016-0368-7

Farr, DF, Rossman, AY. 2016 - Fungal Databases, Systematic Mycology and Microbiology Laboratory, ARS, USDA. from http://nt.ars-grin.gov/fungaldatabases/

Hatakeyama S, Tanaka K, Harada Y 2008 - Bambusicolous fungi in Japan (7): a new coelomycetous genus, Versicolorisporium. Mycoscience 49, 211-214.

Hyde KD, Hongsanan S, Jeewon R, Bhat DJ et al. 2016 - Fungal diversity notes 367-490: taxonomic and phylogenetic contributions to fungal taxa. Fungal Diversity 80, 1-270

Hyde KD, Jones EBG, Liu JK, Ariyawansa H et al. 2013 - Families of Dothideomycetes. Fungal Diversity 63, 1-313.

Index Fungorum 2016 - http://www.indexfungorum.org/Names/IndexFungorumRegister.htm (October, 2016)

Jayasiri SC, Hyde KD, Ariyawansa HA, Bhat J et al. 2015 - The Faces of Fungi database: fungal names linked with morphology, phylogeny and human impacts. Fungal Diversity 74, 118.

Jeewon R, Liew ECY, Hyde KD. 2002 - Phylogenetic relationships of Pestalotiopsis and allied genera inferred from ribosomal DNA sequences and morphological characters. Molecular Phylogenetics and Evolution 25, 378-392. 
Jeewon R, Liew ECY, Simpson, JA, Hodgkiss, IJ, Hyde KD 2003 - Phylogenetic significance of morphological characters in the taxonomy of Pestalotiopsis species. Molecular Phylogenetics and Evolution 27, 372-383.

Jeewon R, Liew ECY, Hyde KD. 2004 - Phylogenetic evaluation of species nomenclature of Pestalotiopsis in relation to host association. Fungal Diversity 17, 39-55.

Jeewon R, Ittoo J, Mahadeb D, Jaufeerally-Fakim Y et al. 2013 - DNA based identification and phylogenetic characterisation of endophytic and saprobic fungi from Antidesma madagascariense, a medicinal plant in Mauritius. Journal of Mycology 2013, 1- 10

Jeewon R, Hyde KD. 2016 - Establishing species boundaries and new taxa: recommendations to resolve taxonomic ambiguities. Mycosphere: in prep

Katoh K, Standley DM. 2013 - MAFFT multiple sequence alignment software version 7: improvements in performance and usability. Journal of Molecular Evolution 30, 772-780.

Kishino H, Hasegawa M 1989 - Evaluation of the maximum likelihood estimate of the evolutionary tree topologies from DNA sequence data, and the branching order in Hominoidea. Journal of Molecular Evolution 29, 170-179.

Liu JK, Phookamsak R, Dai DQ, Tanaka K et al. 2014 - Roussoellaceae, a new pleosporalean family to accommodate the genera Neoroussoella gen. nov., Roussoella and Roussoellopsis. Phytotaxa 181, 1-33.

Liu JK, Phookamsak R, Doilom M, Wikee S et al. 2012 - Towards a natural classification of Botryosphaeriales. Fungal Diversity 57, 149-210.

Liu JK, Hyde KD, Jones EBG, Ariyawansa HA et al. 2015 - Fungal diversity notes 1-110: Taxonomic and phylogenetic contributions to fungal species. Fungal Diversity 72, 1-197.

Liu JK, Phookamsak R, Jones EBG, Zhang Y et al. 2011 - Astrosphaeriella is polyphyletic, with species in Fissuroma gen. nov., and Neoastrosphaeriella gen. nov. Fungal Diversity 51, 135-154.

Liu YJ, Whelen S, Hall BD. 1999 - Phylogenetic relationships among ascomycetes: evidence from an RNA polymerase II subunit. Journal of Molecular Evolution 16, 1799-1808.

Miller MA, Pfeiffer W, Schwartz T. 2010 - Creating the CIPRES Science Gateway for inference of large phylogenetic trees. In Gateway Computing Environments Workshop (GCE) pp. 1-8. IEEE.

Nylander JAA. 2004 - MrModeltest 2.0. Program distributed by the author. Evolutionary Biology Centre, Uppsala University

Paul R, Julie TS. 2003 - "Producing and Consuming Chemicals: The Moral Economy of the American Lawn", Economic Geography 79(4), p. 425-45

Phookamsak R, Liu JK, McKenzie EHC, Manamgoda DS et al. 2014 - Revision of Phaeosphaeriaceae. Fungal Diversity 68, 159-238

Purahong W, Hyde KD. 2011 - Effects of fungal endophytes on grass and non-grass litter decomposition rates. Fungal Diversity 47, 1-7.

Rambaut A, Drummond AJ. 2007 - http://beast. bio. ed. ac. uk/Tracer. Tracer v1, 4. Tamura et al. 2011.

Rambaut A, Drummond AJ. 2008 - FigTree: Tree figure drawing tool, version 1.2. 2. Institute of Evolutionary Biology, University of Edinburgh.

Rannala B, Yang Z. 1996 - Probability distribution of molecular evolutionary trees: a new method of phylogenetic inference. Journal of Molecular Evolution 43, 304-311.

Raven PH, Johnson GB. 1995 - Carol J. Mills, ed. Understanding Biology (3rd ed.). WMC Brown. 536 p. 
Ronquist F, Teslenko M, van der Mark P, Ayres D et al. 2011 - MrBayes 3.2: Efficient Bayesian phylogenetic inference and model choice across a large model space. Systematic Biology 61, 539-542.

Sarwar M H, Sarwar MF, Sarwar M, Qadri NA et al. 2013 - The importance of cereals (Poaceae: Gramineae) nutrition in human health: A review. Journal of Cereals and Oilseeds 4, 3235.

Soreng RJ, Peterson PM, Romschenko K, Davidse G et al. 2015 - "A worldwide phylogenetic classification of the Poaceae (Gramineae)". Journal of Systematics and Evolution 53, $117-137$.

Stamatakis A. 2006 - RAxML-VI-HPC: maximum likelihood-based phylogenetic analyses with thousands of taxa and mixed models. Bioinformatics 22, 2688-2690.

Stamatakis A, Hoover P, Rougemont J. 2008 - A rapid bootstrap algorithm for the RAxML web servers. Systematic Biology 57, 758-771.

Sung GH, Sung JM, Hywel-Jones NL Spatafora JW. 2007 - A multi-gene phylogeny of Clavicipitaceae (Ascomycota, Fungi): Identification of localized incongruence using a combinational bootstrap approach. Molecular Phylogenetics and Evolution 44, 12041223.

Swofford DL. 2002 - PAUP: phylogenetic analysis using parsimony, version 4.0 b10. Sinauer Associates, Sunderland.

Vilgalys R, Hester M. 1990 - Rapid genetic identification and mapping of enzymatically amplified ribosomal DNA from several Cryptococcus species. Journal of Bacteriology 172, 4238-4246.

White TJ, Bruns TD, Lee S, Taylor J. 1990 - Amplification and direct sequencing of fungal ribosomal RNA genes for phylogenetics. PCR Protocols: Guide Methods Application 18, 315-322.

Wijayawardene NN, Crous PW, Kirk PM, Hawksworth DL et al. 2014 - Naming and outline of Dothideomycetes - 2014 including proposals for the protection or suppression of generic names. Fungal Diversity 69, 1-55.

Wong MKM, Hyde KD. 2001 - Diversity of fungi on six species of Gramineae and one species of Cyperaceae in Hong Kong. Mycological Research 105, 1485-1491.

Zhaxybayeva O, Gogarten JP. 2002 - Bootstrap, Bayesian probability and maximum likelihood mapping: exploring new tools for comparative genome analyses. BMC Genomics 3: 4. 